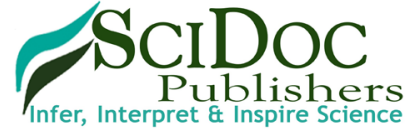

International Journal of Clinical \& Experimental Otolaryngology (IJCEO) ISSN 2572-732X

\title{
A Combined Endolaryngeal Approach for Bilateral Vocal Fold Immobility: Microlaryngoscopic Submucosal Cordotomy and Endo-Extralaryngeal Triple-Suture Lateralization
}

\author{
Case Report
}

Denizoğlu $\dot{\mathrm{I}}^{*}$

Medicalpark Izmir Hospital, Department of Otorhinolaryngology, Turkey.

\section{Abstract}

Bilateral vocal fold immobility (BVFI) is a challenging clinical entity for laryngologists. The voice may be nearly normal, but mostly there is a severe inspiratory deficiency. The patients may need surgical interventions primarily for life-threatening dyspnea. While treating the dyspnea, dysphonia appears to be a problem to a certain extent. It is necessary to maintain a balance between providing a serviceable voice and prevention from further surgery for relapsing dyspnea. In the literature, there are various techniques which have been introduced for the surgical management of BVFI. In this report we present a modified approach performed on a BVFI patient, targeting to balance between dyspnea and dysphonia.

Keywords: Bilateral Vocal Fold Immobility; Posterior Cordotomy; Laterofixation; Inspiratory Stridor.

\section{Introduction}

Because of the complex innervation and re-innervation features of the larynx [1], paralysis of the vocal folds (VFs) is not atypical paralytic condition as seen in extremities. Bilateral laryngeal nerve injury may be a life-threatening clinical entity which may need management under emergency conditions. It is important to make a distinction between cricoarytenoid joint fixation and loss of innervation in order to proceed for treatment bilateral vocal fold immobility (BVFI). Laryngeal electromyography and palpation of the arytenoid may help this distinction. Surgical trauma (i.e. thyroidectomy) is the most common cause of BVFI [2].

Depending on its functional situation, BVFI mostly needs surgical intervention(s) basically starting with a tracheotomy. Voice and breathing are in an inverse relationship in the management of BVFI. From the aerodynamic-biomechanical standpoint, hoarseness seems to be inevitable to some extent in procedures which change the endolaryngeal geometry surgically in BVFI patients. Several methods have been introduced for treatment of BVFI: unilateral total ventriculocordectomy [3], arytenoidectomy $[4,5]$, posterior cordectomy $[6]$ and laterofixation $[7,8]$.
Surgical management of BVFI should be planned to make a critical balance between dyspnea and dysphonia. Mostly, the surgical interventions create irreversible structural and functional alterations to some extent. In case of undesirable results such as overcorrection of the airway and resulting aphonia, it may not be possible to provide a serviceable voice again. In such a situation, tracheotomy with a fenestrated tracheotomy cannula may even be more favorable. Treatment plan then may start from minimally invasive procedures to more invasive and complex procedures.

\section{Case Report}

A fifty-three year old female was referred to our clinical vocology unit with inspiratory stridor. She had been operated for nodular thyroid disease 6 years ago. After total thyroidectomy, her prominent problem has been severe stridor, especially at night while she is sleeping. Her breathing deficiency got worse in the last year and she was not able to manage her daily life which was Grade 4 on MRC Scale (Medical Research Council's Breathlessness Scale [9]).

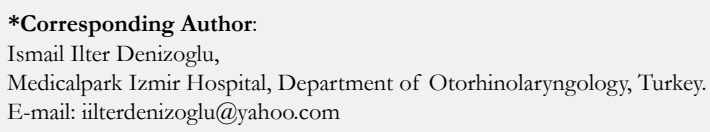

Citation: Denizoğlu II (2017) A Combined Endolaryngeal Approach for Bilateral Vocal Fold Immobility: Microlaryngoscopic Submucosal Cordotomy and Endo-Extralaryngeal TripleSuture Lateralization. Int J Clin Exp Otolaryngol. 3(3), 69-72. doi: http://dx.doi.org/10.19070/2572-732X-1700013 
The patient was hospitalized and a combined operation was performed under general anesthesia. After direct microlaryngoscopy, vocal fold mucosa was incised medially and parallel to the superior arcuate line. Reinke's space was dissected submucosally, extending to the subglottic border of the vocal ligament. The vocal ligament and the thyroarytenoid muscle were truncated from their origin from the arytenoid cartilage. The muscle immediately retracts away to the anterior direction and forms a bulk for future phonation at the anterior glottis. It is important to truncate the muscle with its two bellies (i.e. thyrovocalis and thyromuscularis) with the vocal ligament. Then the mucosal incision was sutured by $8 / 0$ vicryl in order to prevent granuloma formation. The Lichtenberger suture instrument was then used for suture lateralization of the posterior glottis. To decrease the risk of long-term soft tissue loosening, three sutures were placed leaving $2 \mathrm{~mm}$ space in-between. The first suture was placed $2 \mathrm{~mm}$ posteriorly to the tip of vocal process. The second one was placed to the level of the vocal process. These two sutures were aimed to provide an opposite vector to lateral cricoarytenoid (LCA) muscle. The last suture was placed to the posterior membranous glottis for closing the tissue gap which was left by the truncated TA muscle (Figure 1).

Postoperatively, one-year follow-up showed no dyspnea or inspiratory stridor for daily life conditions. Despite a moderate hoarseness (i.e. breathy voice), patient's vocal communication was not affected except in noisy environment. In the early postoperative period, there was a moderate to severe anterior glottic edema but posterior glottis was prominently open. After oral steroid treatment, the edema resolved. After edema was resolved, the MRC scale of breathlessness became grade 1, and the tracheotomy was not necessary for the patient. The mucosal defect was completely healed in the first week and no granuloma formation was determined. Swallowing was not affected, no aspiration was determined possibly because of the arytenoid bulk left on site. At the patient's first-year follow-up evaluation there was not re-narrowing of the posterior glottal gap (Figure. 2).

Preoperatively, maximum phonation time was calculated as 3 seconds, it decreased to 2 seconds in postoperative assessment. Interestingly, functional $(\mathrm{F})$, physical $(\mathrm{P})$, and emotional $(\mathrm{E})$ parameters in Voice Handicap Index (VHI) showed remarkable improvement. Preoperative VHI results were F17, P19, and E15. Postoperative findings were F9, P7, and E5. It was possibly due to deleterious effects of preoperative dyspnea to communicative skills.

\section{Discussion}

Overall success rate for surgical management of BVFI depends on voice and breathing outcome. Various techniques have been described to accomplish a balance between, these, two important quality of life factors. The larynx is a complicated valve system and functions of the larynx are basically executed by that active valve mechanism. Vocal folds function as unidirectional valves which resist airflow from outside-in. On the other hand, the ventricular folds (i.e. False vocal folds) work oppositely as a unidirectional valve resisting airflow inside-out direction in order to help for thoracic fixation function of larynx (Figure 3).

True VFs form an inside-out valve from the aerodynamic and biomechanical approach which works properly for phonation in BVFI but when it comes to breathing, the valve function leads to an inspiration deficiency. The possible solution lies behind muscular vectors and the functional dominance in glottis. The anterior two-thirds of the glottis primarily comprise the phonatory function whereas the posterior one-third serves as the dominant component of the respiratory function of the glottic space. Thus, in order to keep inspiration-phonation-aspiration in balance; three procedural aspects should be taken into consideration:

1. A lateral movement of the vocal process in the opposite direction of LCA muscle by suture lateralization. This was done with lateralization sutures which pass around the vocal process of the arytenoid cartilage.

2. Keeping the glottic valve function at the anterior part of glottis for providing mucosal vibration or at least falsetto effect. The vocal ligament and thyroarytenoid muscle with its two bellies were truncated without excision. The body of the muscle was retracted anteriorly to make a narrower anterior glottic space.

3. Prevention of the aspiration by leaving the arytenoid bulk on site.

Figure 1. The operation procedure. Upper line from left: 1: Superolateral incision of vocal fold mucosa and dissection of mucosa to the inferior border of vocal ligament. 2: Truncation of vocal ligament and TA muscle until the muscle is drawn away anteriorly. 3: Suturing the mucosa with $8 / 0$ vicryl. 4,5,6: Suture laterofixation by Lichtenberger's needle carrier. The first suture was placed $2 \mathrm{~mm}$ anteriorly to the vocal process. Others were placed posteriorly to the first suture.
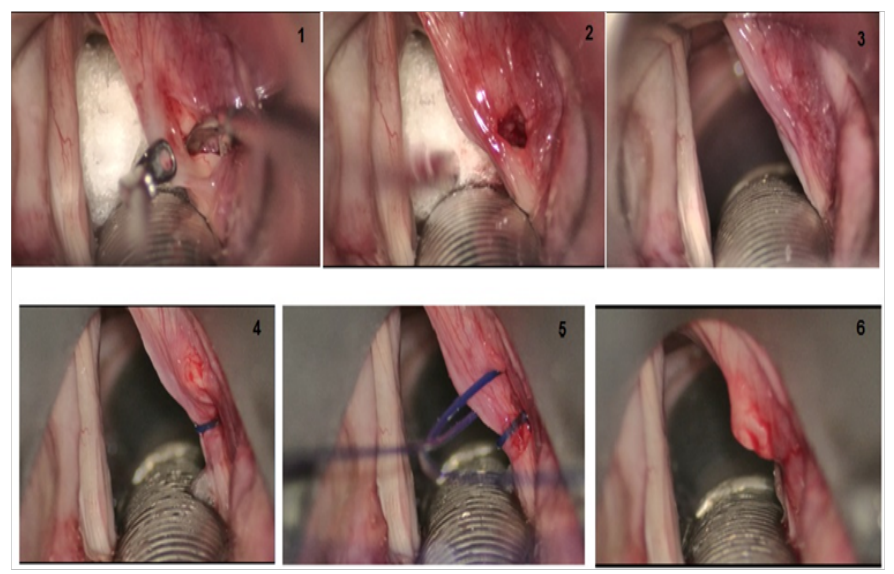
Figure 2. The preoperative and postoperative (1st year) endoscopic views of the BVFI patient.

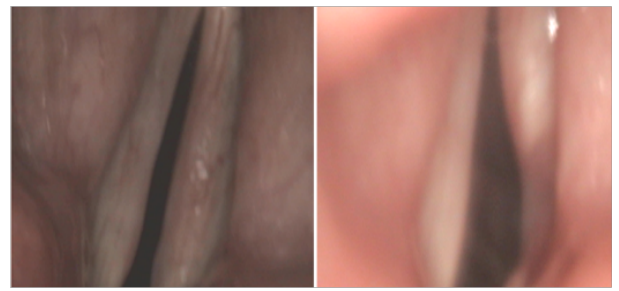

Figure 3. Schematic representation of double-valve function of larynx. FVF: False Vocal Fold, VF: Vocal Fold, arrows point the direction of airflow.
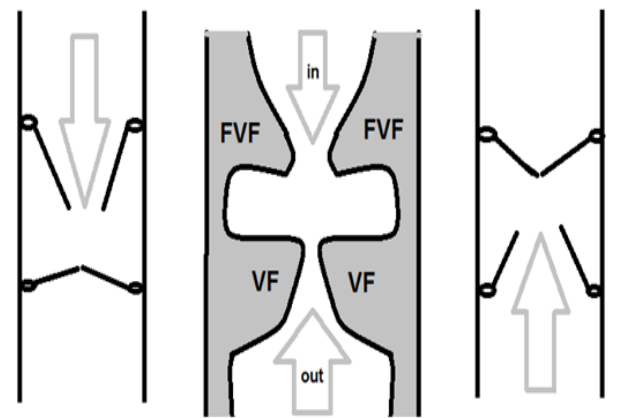

Vocal fold lateralization is a simple procedure especially if it is performed by an endo-extralaryngeal needle carrier. The needle carrier helps to place the suture to an exact point inside the larynx with a single attempt which also minimizes edema and hematoma in laryngeal tissues. Even under the best of circumstances, suture lateralization may result in a severe edema of the lateralized VF and tracheostomy may be necessary in the first week of suture lateralization surgery. We used Lichtenberger's needle carrier in our patient, a meticulous dissection was made to minimize the tissue reaction. No hematoma was observed in postoperative 3 days. Patient has had a moderate dyspnea at the postoperative $5^{\text {th }}$ day because of the edema at the operated side. She was relieved after a short course of intravenous steroid treatment and no tracheotomy was needed.

Long-term results of suture lateralization alone may not provide enough airway possibly because the thread is loosened in time by cutting the soft tissues. Fibrosis in the tissue then may not be enough for a lateralization effect. In order to enhance this effect, lateralization of true and false vocal folds by crossing sutures has been introduced by Katilmis et.al., [10]. Because the valve function of the ventricular folds are against expiration (not inspiration), ventricular folds were not included in lateralization procedure in our patient.

Arytenoidectomy, posterior cordectomy/cordotomy (even assisted by laser) on the other hand, may result in granuloma or hypertrophic scar formation which, in advance, may lead to inspiratory stridor [11-13]. In order to prevent revision surgeries due to persistent airway symptoms (re-narrowing scar tissue, granuloma, and tissue loosening after the single suture lateralization), we used three sutures to minimize the long-term loosening of the tissues and combined microlaryngeal posterior submucosal cordotomy with suture lateralization for a greater space posteriorly. One year follow-up did not show tissue reactions such as the granuloma or hypertrophic cicatrix and airway obstruction as well. The retracted vocal ligament with the muscle was still obvious at the anterior vocal fold.

\section{Conclusion}

Microlaryngoscopic submucosal cordotomy combined with endolaryngeal triple suture revealed a favorable long-term follow-up in a BVFI patient. The submucosal cordotomy with minimally invasive dissection prevented further re-narrowing of the posterior glottic region by hypertrophic scar and granuloma formation. Triple suturing (one of which passes around the vocal process) provided effective lateral movement of the vocal process. No aspiration was observed and tracheotomy was not needed after the operation. Among various surgical methods for management of BVFI, this new modification seems to provide an effective balance for laryngeal functions. Further studies on large patient groups are needed to confirm the effectiveness of the method.

\section{References}

[1]. Crumley RL (2000) Laryngeal synkinesis revisited. Ann Otol Rhinol Laryngol. 109(4): 365-371.

[2]. Rosenthal LH, Benninger MS, Deeb RH (2007) Vocal fold immobility: a longitudinal analysis of etiology over 20 years. Laryngoscope. 117(10): 1864-1870.

[3]. Jackson C (1922) Ventriculocordectomy: A new operation for the cure of goitrous glottic stenosis. Arch Surg. 4: 257-274.

[4]. Woodman D (1946) A modification of the extralaryngeal approach to arytenoidectomy for bilateral abductor paralysis. Arch Otolaryngol. 43: 63-65.

[5]. Thornell WC (1948) Intralaryngeal approach for arytenoidectomy in bilateral vocal cord paralysis. Arch Otolaryngol. 47(4): 505-508.

[6]. Kirchner FR (1979) Endoscopic lateralization of the vocal cord in abductor paralysis of the larynx. Laryngoscope. 89(11): 1779-1783. DOI: 10.1288/00005537-197911000-00010

[7]. Ejnell H, Mansson I, Hallén O, Bake B, Stenborg R, et al., (1984) A simple operation for bilateral vocal cord paralysis. Laryngoscope. 94(7): 954-958.

[8]. Lichtenberger G (1983) The endo-extralaryngeal needle carrier instrument. Laryngoscope. 93(10): 1348-1350.

[9]. Fletcher CM (1952) The clinical diagnosis of pulmonary emphysema; an experimental study. Proc R Soc Med. 45(9): 577-584.

[10]. Katilmis H, Ozturkcan S, Basoglu S, Aslan H, Ilknur AE, et al., (2011) New technique for the treatment of bilateral vocal cord paralysis: Vo- 
cal and ventricular fold lateralization using crossing sutures with thyroplasty technique. Acta Oto-Laryngologica. 131(3): 303-309. DOI: 10.3109/00016489.2010.526143.

[11]. Özdemir S, Tuncer Ü, Tarkan Ö, Kara K, Sürmelioğlu Ö (2013) Carbon dioxide laser endoscopic posterior cordotomy technique for bilateral abductor vocal cord paralysis: a 15-year experience. JAMA Otolaryngol Head Neck Surg. 139(4): 401-404. DOI: 10.1001/jamaoto.2013.41
[12]. Khalil MA, Abdel Tawab HM (2014) Laser Posterior Cordotomy: Is it a Good Choice in Treating Bilateral Vocal Fold Abductor Paralysis? Clin Med Insights Ear Nose Throat. 7: 13-17. DOI:10.4137/CMENT.S15888

[13]. Rinne J (1991) Late results of laterofixation in the treatment of bilateral abductor paralysis of the vocal cords: A clinical study with long-term followup. Clin Otolaryngol Allied Sci. 16(5): 436-441. 